Math. Model. Nat. Phenom.

Vol. 5, No. 5, 2010, pp. 46-63

DOI: $10.1051 / \mathrm{mmnp} / 20105504$

\title{
Waves of Autocrine Signaling in Patterned Epithelia
}

\author{
C. B. Muratov ${ }^{1, *}$ and S. Y. Shvartsman ${ }^{2}$ \\ ${ }^{1}$ Department of Mathematical Sciences, New Jersey Institute of Technology \\ Newark, NJ 07102, USA \\ ${ }^{2}$ Department of Chemical Engineering and Lewis Sigler Institute for Integrative Genomics \\ Princeton University, Princeton, NJ 08544, USA
}

\begin{abstract}
A biophysical model describing long-range cell-to-cell communication by a diffusible signal mediated by autocrine loops in developing epithelia in the presence of a morphogenetic prepattern is introduced. Under a number of approximations, the model reduces to a particular kind of bistable reaction-diffusion equation with strong heterogeneity. In the case of the heterogeneity in the form of a long strip a detailed analysis of signal propagation is possible, using a variational approach. It is shown that under a number of assumptions which can be easily verified for particular sets of model parameters, the equation admits a unique (up to translations) variational traveling wave solution. A global bifurcation structure of these solutions is investigated in a number of particular cases. It is demonstrated that the considered setting may provide a robust developmental regulatory mechanism for delivering chemical signals across large distances in developing epithelia.
\end{abstract}

Key words: cell communication, front propagation, traveling waves

AMS subject classification: $35 \mathrm{~K} 55$, 35J60, 35J20, 35K57

\section{Introduction}

Embryonic development requires tight spatial control of cell differentiation [16]. One of the most basic mechanisms for accomplishing this task relies on the establishment of morphogen gradients in developing epithelia. Morphogen gradients are concentration profiles of proteins that provide

${ }^{*}$ Corresponding author. E-mail: muratov@ njit.edu 
dose-dependent control of gene expression [24, 2]. Given the low rates of protein diffusion in tissues, direct action of morphogen gradients is restricted to a small number of cells. Larger territories can be patterned by cellular relays, where one short-ranged molecule induces the production of a secondary signal that diffuses a little further, and so on.

In an important version of this mechanism, cells respond to a diffusible signal by releasing more of the same signaling molecule [9]. The resulting positive feedback loops, termed autocrine loops, can give rise to waves of signaling that propagate over large distances, coordinating differentiation of dozens and even hundreds of cells. A remarkable example of this effect is the morphogenetic furrow, a traveling wave of cell differentiation that gives rise to a periodic arrangement of Drosophila photoreceptors [11]. This wave is generated by a positive feedback loop formed by two diffusible proteins.

In recent work, we have analyzed a number of biophysical models giving rise to front propagation in autocrine relays $[20,22,21,19]$. There we treated an idealized situation in which the epithelium is considered as a flat homogeneous sheet of cells of infinite extent. At the same time, it is very common in development that cell differentiation is punctuated by a sequence of welldefined patterning events $[29,13,31]$. In this situation each subsequent step of differentiation proceeds in the gene expression background established during the preceding step. This makes it necessary within developmental contexts to analyze the problem of signal propagation in strongly heterogeneous media.

Here we investigate a situation in which autocrine signals act on fields of cells that have been patterned by previous rounds of signaling and are not necessarily uniform [9]. Specifically, we will look into a particular case of stratified epithelia, i.e. the epithelia in which the morphogenetic prepattern has the form of long stripes, and study the question of front propagation along the stripes from a localized stimulus. Mathematically, this setting offers a considerable simplification, since front propagation in such a problem is closely related to the problem of existence of traveling wave solutions. In contrast, in more complicated patterned epithelia one would need to work with the concept of generalized traveling waves (see e.g. [3, 30]). Furthermore, this setting falls within the scope of the variational approach to front propagation problems developed recently in $[17,14,18]$.

Our paper is organized as follows. In Sec. 2., we formulate a biophysical model of patterned epithelia with an autocrine feedback loop and perform suitable model reductions to make it amenable to analysis. In Sec. 3., we extend the variational approach of [18] to the problem under consideration and study existence and uniqueness of traveling wave solutions in the case where autocrine signaling occurs only in a single narrow strip of cells. The main results of this section are contained in Theorems 1 and 3 of that section. Then, in Sec. 4. we consider a few specific examples in which the assumptions of the theorems my be verified and present some numerical results. Finally, in Sec. 5. we summarize our findings and discuss some directions for future research. 


\section{Model}

Let us begin by formulating the mechanistic model of an autocrine relay which takes into account the underlying heterogeneity of the gene expression pattern present at a given developmental stage. For simplicity, we will consider a situation in which only a single signaling molecule is involved in signal transmission along the epithelium. This signaling molecule (ligand) is subject to the following elementary processes: it can diffuse in the thin extracellular space adjacent to the epithelium, it can reversibly bind to its specific cell surface receptor, and it can be secreted by cells into the extracellular space. The secretion rate is further assumed to be proportional to the density of a ligand-processing intracellular species. Importantly, a positive feedback is assumed to exist, via the mechanism of ligand-induced ligand release, whereby the production of the ligand-processing intracellular molecules is autocatalytic and is upregulated by an increase in the number of the signaling molecules bound to their cell surface receptors [22, 21, 20].

To introduce heterogeneity into this problem, we assume that only certain groups of cells express the extracellular species mediating the positive autocrine feedback loop. Then, within the continuum modeling framework, the biophysical processes discussed above can be recast into the following system of equations:

$$
\begin{aligned}
\frac{\partial S}{\partial t} & =D_{s}\left(\frac{\partial^{2} S}{\partial x^{2}}+\frac{\partial^{2} S}{\partial y^{2}}\right)-k_{\mathrm{on}} R S+k_{\mathrm{off}} C+g_{r} P+G_{0}(x, y, t) \\
\frac{\partial C}{\partial t} & =k_{\mathrm{on}} R S-\left(k_{\mathrm{off}}+k_{\mathrm{ec}}\right) C, \\
\frac{\partial P}{\partial t} & =-k_{p} P+g_{p} \sigma\left(C / C_{T}\right) \chi_{p}(x, y) .
\end{aligned}
$$

Here, $S$ denotes the concentration of the signaling molecule in the thin extracellular layer above the epithelial cells, $P$ is the density of the ligand-processing intracellular species, and $C$ is the density of the ligand-receptor complexes. In (2.1), $D_{s}$ is the effective diffusion constant of the signaling molecule, $R$ is the receptor density (receptors are assumed to be in excess), $k_{\text {on }}$ and $k_{\text {off }}$ are the binding and dissociation constants between the signaling molecule and the receptor, respectively, and $g_{r} P$ is the secretion rate, with $g_{r}$ the proportionality constant. Thus, the secretion rate in (2.1) is assumed to be proportional to the density of the ligand-processing species $P$. Furthermore, (2.2) describes the dynamics of the ligand-receptor complexes and also takes into account signalinduced endocytosis, whose rate constants is given by $k_{\mathrm{ec}}$. Finally, (2.3) contains the first-order degradation term with the rate $k_{p}$, as well as the sigmoidal dependence of the production rate on the density of complexes $C$, with the threshold $C_{T}$ and the maximum production rate $g_{p}$. Also in (2.3), the function $\chi_{p}(x, y)$ defines the cells in which the ligand-processing intracellular species is expressed (present), i.e., it is equal to 1 in those cells in which $P$ is present and 0 where it is absent; and $G_{0}(x, y, t)$ denotes a positive transient perturbation (by some other intracellular species from an earlier patterning step) which has the effect of signal initiation.

The model just introduced consists of a nonlinear system of coupled partial and ordinary differential equations with spatially varying parameters, and as such is rather difficult to analyze. In the following, we introduce a biophysically-motivated simplification, which has to do with the fact 
that the receptor binding dynamics typically occurs on a time scale much shorter than the times scale of the patterning events $[22,28]$. This results in a steady-state approximation for $(2.2)$. To further simplify the analysis, we also introduce a steady-state approximation into (2.3). After a little algebra equations $(2.1)-(2.3)$ reduce to a single equation

$$
\frac{\partial S}{\partial t}=D_{s}\left(\frac{\partial^{2} S}{\partial x^{2}}+\frac{\partial^{2} S}{\partial y^{2}}\right)-k_{s} S+g_{s} \sigma\left(S / S_{T}\right) \chi_{p}(x, y)+g_{0}(x, y, t),
$$

where we introduced new parameters

$$
k_{s}=\frac{k_{\mathrm{on}} k_{\mathrm{ec}} R}{k_{\mathrm{off}}+k_{\mathrm{ec}}}, \quad g_{s}=\frac{g_{r} g_{p}}{k_{p}}, \quad S_{T}=\frac{k_{\mathrm{off}}+k_{\mathrm{ec}}}{k_{\mathrm{on}} R} C_{T},
$$

and $g_{0}$ is a suitably rescaled version of $G_{0}$.

Note that the obtained reduced model in (2.4) shares many common features with the classical models in combustion theory and population biology (see e.g. [4, 23, 30]). In particular, from dimensional analysis the diffusion length $L=\sqrt{D_{s} / k_{s}}$ naturally emerges. In the developmental context this length (typically on the order of a few cell diameters) is expected to be of the same order as the characteristic size of the morphogenetic pre-pattern. Therefore, a question of particular interest is the interplay between the diffusion and degradation terms in (2.4), setting the diffusion length scale, with the nonlinear reaction term which is localized in the heterogeneities. In the following, we investigate this interplay in a specific heterogeneous setting via a rigorous analysis of existence and multiplicity of traveling wave solutions.

\section{Traveling waves}

We now consider a particular situation in which the epithelium is considered to be a half-plane, with the ligand-producing cells forming a semi-infinite strip normal to the reflecting boundary (Figure 1). Without any loss of generality, we can further perform an even extension of the problem to the whole of $\mathbb{R}^{2}$. Then, after introducing a non-dimensionalization

$$
S=\frac{g_{s}}{k_{s}} u, \quad x \rightarrow \sqrt{\frac{D_{s}}{k_{s}}} x, \quad t \rightarrow k_{s}^{-1} t, \quad S_{T}=\frac{g_{s}}{k_{s}} a .
$$

we obtain a parabolic problem

$$
u_{t}=\Delta u-u+\sigma_{\nu, a}(u) \chi_{[-l, l]}(y)+g(x, y, t),
$$

for the quantity $u: \mathbb{R}^{2} \times[0, \infty) \rightarrow[0,1]$, with zero initial data. Here

$$
\sigma_{\nu, a}(u)=\frac{u^{\nu}}{a^{\nu}+u^{\nu}}, \quad \chi_{[-l, l]}(y)= \begin{cases}1, & |y| \leq l \\ 0, & |y|>l\end{cases}
$$




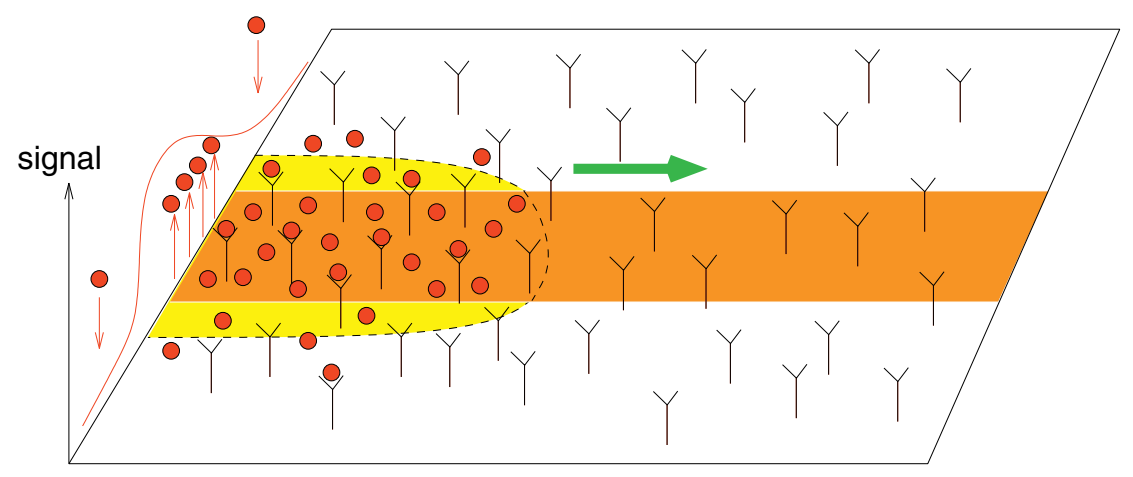

Figure 1: The schematics of switching on of an autocrine loop in a row of cells expressing a ligandprocessing protease. The ligand is secreted only in a narrow strip of the epithelium, but is degraded upon binding to receptors which are uniformly distributed throughout the epithelium. The pattern is initiated by a localized input at the left end of the epithelium and propagates to the right. The dashed line shows an instantaneous level set of the ligand concentration at the cell surfaces.

and $g(x, y, t) \in C_{0}^{\infty}\left(\mathbb{R}^{2} \times[0, \infty)\right)$ is a given non-negative function (here and below $C_{0}^{\infty}$ denotes smooth functions with compact support). In particular, we assumed that the sigmoidal function in (3.2) is given by a Hill function $\sigma_{\nu, a}(u)$ with the Hill coefficient $\nu$ and the threshold $a$, which is a common modeling assumption in the studies of regulatory networks [25].

Note that since the nonlinear term in (3.2) is uniformly Lipschitz in $u$ and since $u=0$ and $u=1+\max g$ are a sub- and a super-solution, respectively, by standard parabolic theory [15] we get global existence of weak solutions of (3.2). In particular, these solutions decay superexponentially as $|x|,|y| \rightarrow \infty$ and, hence, lie in the exponentially weighted Sobolev spaces $H_{c}^{1}\left(\mathbb{R}^{2}\right)$ for each $t \geq 0$ and every $c>0$. Here, as usual [18], $H_{c}^{1}\left(\mathbb{R}^{2}\right)$ denotes the Hilbert space obtained as a closure of $C_{0}^{\infty}\left(\mathbb{R}^{2}\right)$ with respect to the norm defined by

$$
\|u\|_{H_{c}^{1}\left(\mathbb{R}^{2}\right)}^{2}=\|u\|_{L_{c}^{2}\left(\mathbb{R}^{2}\right)}^{2}+\|\nabla u\|_{L_{c}^{2}\left(\mathbb{R}^{2}\right)}^{2}, \quad\|u\|_{L_{c}^{2}\left(\mathbb{R}^{2}\right)}^{2}=\int_{\mathbb{R}^{2}} e^{c x}|u|^{2} d x d y .
$$

Following the transient perturbation specified by $g$, the signal distribution $u$ will solve (3.2) with $g=0$ for $t \geq T$, for some $T>0$. In other words, for $t \geq T$ the problem for $u$ becomes equivalent to an initial-value problem specified by (3.2) with $g \equiv 0$ and positive initial data $u_{0}(x, y)=u(x, y, T) \in H_{c}^{1}\left(\mathbb{R}^{2}\right)$ with arbitrary $c>0$. As is well known in this general class of models (see e.g. [8]), depending on the amplitude and size of $u_{0}$, as well as on the values of $\nu, a$, and $l$, one would expect an initiation of a propagating front of signaling. Fronts are traveling wave solutions of (3.2) which invade the stable equilibrium $u=0$ from left to right with some speed $c>0$. We note that in the present context the relevant class of traveling wave solutions are the so-called variational traveling waves $[17,14,18]$, namely functions $\bar{u} \in H_{c}^{1}\left(\mathbb{R}^{2}\right)$ solving

$$
\bar{u}_{x x}+\bar{u}_{y y}+c \bar{u}_{x}-\bar{u}+\sigma_{\nu, a}(\bar{u}) \chi_{[-l, l]}(y)=0 \quad \text { weakly in } H_{c}^{1}\left(\mathbb{R}^{2}\right) .
$$


Indeed, the ability of solutions of equations like (3.2) with the initial data decaying sufficiently fast at $x=+\infty$ to propagate towards $x=+\infty$ has been shown to depend critically on existence of minimizers for the functional

$$
\Phi_{c}[u]=\int_{\mathbb{R}^{2}} e^{c x}\left(\frac{1}{2}|\nabla u|^{2}+V(u, y)\right) d x d y,
$$

where for our system

$$
V(u, y)=\frac{u^{2}}{2}-\chi_{[-l, l]}(y) \int_{0}^{u} \sigma_{\nu, a}(s) d s,
$$

over all functions $u \in H_{c}^{1}\left(\mathbb{R}^{2}\right)$ and $c>0[17,14,18]$. In particular, for a wide class of wave-like solutions, i.e. solutions of (3.2) on which $\Phi_{c}[u(\cdot, t)]<0$ for some $c>0$ and $t \geq T$ only the variational traveling waves can be the long-time attractors of the dynamics in the reference frame associated with the leading edge [17, Theorem 4.11]. Therefore, here we will pay special attention to the study of existence of variational traveling waves in the considered problem. In particular, we will be interested in the question of multiplicity of these solutions.

By energy arguments [14, Proposition 6.6], one would expect the solutions of (3.5) to approach an $x$-independent equilibrium of (3.2), i.e. one expects $\bar{u}(x, y) \rightarrow v(y)$ as $x \rightarrow-\infty$ uniformly in $y$, where $v$ solves the ordinary differential equation

$$
\frac{d^{2} v}{d y^{2}}-v+\sigma_{\nu, a}(v) \chi_{[-l, l]}(y)=0 .
$$

Observe that every solution $v \in H^{1}(\mathbb{R})$ of (3.8) is a critical point of the energy

$$
E[v]=\int_{-\infty}^{+\infty}\left\{\frac{1}{2}\left(\frac{d v}{d y}\right)^{2}+V(v, y)\right\} d y .
$$

In fact, every bounded solution of (3.8) is automatically in $H^{1}(\mathbb{R})$, since the equation becomes linear for $|y|>l$ and, therefore, every bounded solution of (3.8) is explicitly given by

$$
v(y)= \begin{cases}v_{+} e^{l-y}, & y>l \\ v_{-} e^{l+y}, & y<-l\end{cases}
$$

for some constants $v_{ \pm}>0$. Since the energy $E$ is lower-semicontinuous with respect to the weak convergence in $H^{1}(\mathbb{R})$ (see, e.g., [7]), existence of a non-trivial solution for (3.8) is equivalent to existence of a non-trivial minimizer of $E$.

Note that since $v=0$ is always a critical point of $E$, existence of a non-trivial minimizer of $E$ is guaranteed by the condition

$$
\min _{v \in H^{1}(\mathbb{R})} E[v]<0
$$


In fact, (3.11) is also a necessary condition for existence of non-trivial solutions of (3.5) with $c>0$. Indeed, every non-trivial solution of (3.5) is a critical point of $\Phi_{c}$ in $H_{c}^{1}\left(\mathbb{R}^{2}\right)$. Hence, we must have $\Phi_{c}[\bar{u}]=0$ [17, Proposition 3.3]. But this is impossible, if (3.11) does not hold, since in this case by Poincaré inequality [18, Lemma 2.2] we have $\Phi_{c}[\bar{u}] \geq \frac{c^{2}}{8} \int_{\mathbb{R}^{2}} \bar{u}^{2} d x d y>0$. Furthermore, (3.11) cannot be satisfied for any $l>0$, if the value of $a$ is sufficiently large. Indeed, since min $E$ is a monotonically decreasing function of $l$ and a monotonically increasing function of $a$, for (3.11) to hold we must have $a<a_{0}$, where

$$
a_{0}=\sup \left\{a>0: \min _{u \in[0,1]}\left(\frac{u^{2}}{2}-\int_{0}^{u} \sigma_{\nu, a}(s) d s\right)<0\right\} .
$$

The value of $a_{0}$ is the well-known propagation threshold for fronts in the case of $l=\infty[8,1]$.

Our main result concerning variational traveling waves stated below is a natural extension of the results of [18]. Let us note that the main point of the theorem is the statement about uniqueness of the variational traveling waves, which relies on uniqueness of positive equilibria of $E$ with negative energy. We point out that in our setting this assumption can be straightforwardly verified numerically for any set of $a, \nu$ and $l$ (see Theorem 3). In this sense the behavior of our system is expected to be very similar to that of one-dimensional reaction-diffusion equations with a bistable nonlinearity [8]. Nevertheless, because of technical difficulties we were not able to prove this result in full generality.

Theorem 1. Assume there exists a unique solution $v$ of (3.8) for which $E[v]<0$. Then there exists a unique (up to translations in the $x$-direction) solution $\bar{u}$ of (3.5) for a unique value of $c=c^{\dagger}>0$. Furthermore, $\bar{u}$ is a strictly monotonically decreasing function of $x$ converging exponentially to zero as $x \rightarrow+\infty$ and $|y| \rightarrow \infty$, and $u(x, \cdot) \rightarrow v$ uniformly as $x \rightarrow-\infty$, and $\bar{u}(x, y)=\bar{u}(x,-y)$.

Outline of Proof. The proof of our result relies on the construction of minimizers of $\Phi_{c}$ performed in $[14,18]$. Since the arguments of the proofs closely follow those of [18], we will only present the necessary modifications for the considered problem. These are mainly related to the need to consider the solutions of (3.5) on the whole of $\mathbb{R}^{2}$.

As was already noted, minimizers of $\Phi_{c}$ are weak solutions of (3.5). Moreover, by the truncation argument of [14, Proposition 3.3(i)] we have $0 \leq u \leq 1$, and hence by standard elliptic regularity theory [10] every minimizer $u$ is a classical solution outside the lines $y= \pm l$, and is continuous together with its first derivatives in the whole of $\mathbb{R}^{2}$. Also, by the same argument as in [14, Proposition 3.3(iii)], these solutions decay exponentially uniformly as $x \rightarrow+\infty$. At the same time, since $e^{l-|y|}$ is a super-solution for (3.5) for $|y|>l$, by maximum principle we also get the exponential decay of $\bar{u}$ as $|y| \rightarrow \infty$.

To prove existence of minimizers of $\Phi_{c}$, we need to establish lower-semicontinuity of $\Phi_{c}$ with respect to the weak convergence in $H_{c}^{1}\left(\mathbb{R}^{2}\right)$. This result easily follows by noting that the support of the second term in the definition of $V$ is bounded in $y$. Hence, all the arguments of [14] carry over. Similarly, the sublevel sets $\Phi_{c}[u] \leq 0$ of the functional $\Phi_{c}$ subject to the constraint $\int_{\mathbb{R}^{2}} e^{c x}\left|u_{x}\right|^{2} d x d y=$ const are relatively compact in $H_{c}^{1}\left(\mathbb{R}^{2}\right)$. So, existence of minimizers for a unique value of $c=c^{\dagger}>0$ follows as in [18, Theorem 3.9]. Similarly, uniqueness and monotonicity of minimizers follows as in [18, Theorem 3.3], applying strong maximum principle separately 
for $|y|<l$ and $|y|>l$, together with Hopf lemma at $|y|=l$. By monotonicity and exponential decay as $|y| \rightarrow \infty$, we obtain, in turn, that $\bar{u}(x, y) \rightarrow v(y)$ uniformly in $y$ as $x \rightarrow-\infty$. Furthermore, again, from the exponential bound on $\bar{u}$ for large $|y|$ and the arguments of [18, Theorem 3.3(ii)] we conclude that $v$ is a critical point of $E$, such that $E[v]<0$ (unique by assumption).

To prove uniqueness of variational traveling waves, we first note that for $c=c^{\dagger}$ every such solution is a minimizer and, hence, is unique up to translations in the $x$-direction. On the other hand, if $c>c^{\dagger}$, then non-existence follows from [18, Proposition 3.6]. Alternatively, if there exists a variational traveling wave $u$ for some $0<c<c^{\dagger}$, then by [14, Corollary 6.8] we have $u(x, \cdot) \rightarrow v$ uniformly as $x \rightarrow-\infty$. On the other hand, this is clearly impossible in view of existence of a subsolution of (3.2) lying below $u$ and moving with speed $c^{\dagger}-\varepsilon$ for an arbitrary $\varepsilon>0$ (see the proof of [18, Proposition 5.5]).

Finally, symmetry of the solution $\bar{u}$ with respect to the $y=0$ line follows, e.g., by comparing $\Phi_{c}[\bar{u}]$ with the values of $\Phi_{c}$ on the even extensions of $\bar{u}(x, y)$ and $\bar{u}(x,-y)$ from $y>0$ to the whole of $\mathbb{R}^{2}$.

Importantly, the variational characterization of the solutions obtained in Theorem 1 also allows to efficiently obtain upper and lower bounds on the propagation speed $c^{\dagger}$ of the traveling wave $[17,14,18]$ :

Remark 2. Under the assumptions of Theorem 1, let $c_{\min }$ be such that $\Phi_{c_{\min }}[u]<0$ for some $u \in H_{c_{\min }}^{1}\left(\mathbb{R}^{2}\right)$, and let $c_{\max }$ be such that $\Phi_{c_{\max }}[u]=0$ implies $u=0$ for all $u \in H_{c_{\max }}^{1}\left(\mathbb{R}^{2}\right)$. Then $c_{\min }<c^{\dagger}<c_{\max }$.

Let us also note that one would actually expect that all traveling wave solutions for (3.2) are, in fact, variational. The latter is certainly the case, if the problem were considered on a strip rather than the whole of $\mathbb{R}^{2}$ [18, Proposition 3.7]. To prove this statement, however, one would need to undertake a careful analysis of the asymptotic behavior of solutions as $x \rightarrow \pm \infty$ on an unbounded domain.

We now state a result which allows to conveniently characterize all non-trivial critical points of (3.9) as members of a one-parameter family of functions.

Theorem 3. For every $\nu>1$ and every $0<a<a_{0}$, where $a_{0}$ is defined in (3.12), there exist constants $0<v_{1}<v_{2}<1$ such that for every $v_{0} \in\left(v_{1}, v_{2}\right)$ there exists a unique value of $l>0$ for which (3.8) has a positive solution with $v(0)=v_{0}$ and $v( \pm \infty)=0$. Every positive solution of (3.8) is symmetric with respect to $y=0$ and belongs to this class.

Proof. To characterize all the critical points of $E$, we start by multiplying (3.8), as usual, by $d v / d y$ and integrating from $-l$ to $y$. With the help of (3.10) one obtains

$$
\left(\frac{d v}{d y}\right)^{2}-v^{2}+G(v)=G\left(v_{-}\right), \quad G(v)=2 \int_{0}^{v} \sigma_{\nu, a}(s) d s,
$$

where $v_{ \pm}$are defined in (3.10). Also, from (3.10) and (3.13) with $y=l$ we observe that, in fact, $G\left(v_{-}\right)=G\left(v_{+}\right)$. Since, in turn, $G(v)$ is a strictly monotonically increasing function of $v$, we actually have $v_{+}=v_{-}$. 
Let us now turn to the behavior of positive solutions of (3.8) for $-l<y<l$. We claim that the solution is in fact an even function which is monotonically increasing for $y<0$ and decreasing for $y>0$. If not, then by symmetry of $v$ with respect to the point where it reaches its maximum we must have $v\left(y_{0}\right)=v_{+}$for some $-l<y_{0}<l$ and without loss of generality we may assume that $v(y)>v_{+}$for all $-l<y<y_{0}$. But since $G(v)<G\left(v_{+}\right)$for all $v<v_{+}$, from (3.13) we obtain that $d v / d y<-v$ for all $y>y_{0}$, so $v(y)<v_{+}$for these values of $y$. This, once again, contradicts the condition that $v(l)=v_{+}$.

Now, integrating (3.13) and taking the symmetry of solutions into account, we obtain an implicit equation for $v_{-}$:

$$
l=\int_{v_{-}}^{v_{0}} \frac{d s}{\sqrt{G\left(v_{-}\right)-G(s)+s^{2}}}, \quad G\left(v_{0}\right)-v_{0}^{2}=G\left(v_{-}\right),
$$

where we introduced $v_{0}=v(0)$ and took into account (3.10). Thus, all positive solutions of (3.8) can be expressed as a one-parameter family, parametrized by $v_{0} \in\left(v_{1}, v_{2}\right)$, where $v_{1}$ is the first positive root of $G(v)-v^{2}=0$, and $v_{2}>v_{1}$ is the root of $G^{\prime}(v)-2 v=0$ (by maximum principle we have $v<v_{2}$ for any solution of (3.8)). In view of monotonicity of $G(v)-v^{2}$ on $\left(v_{1}, v_{2}\right)$, for each $v_{0} \in\left(v_{1}, v_{2}\right)$, there exists a unique value of $0<v_{-}<v_{0}$ for which the second of (3.14) holds and, hence, a unique value of $l>0$ for which a bounded positive solution of (3.8) exists.

Let us also give an expression for the energy $E$ of a solution of (3.8) in terms of integrals similar to those introduced in (3.14). Using (3.10) and substituting (3.13) into (3.9), after a little algebra we obtain that for a solution characterized by $v_{0}$ in Theorem 3 it holds

$$
E[v]=2 \int_{v_{-}}^{v_{0}} \sqrt{G\left(v_{-}\right)-G(s)+s^{2}} d s-G\left(v_{-}\right) l+v_{-}^{2} .
$$

Thus, establishing existence and uniqueness of bounded positive solutions of (3.8) with negative energy amounts to studying the dependence $l\left(v_{0}\right)$ defined in (3.14) and evaluating the sign of the right-hand side of (3.15) as a function of $v_{0}$. While this dependence is difficult to study analytically, it is a straightforward exercise to evaluate the necessary integrals numerically. We will consider a few specific examples of such a calculation in the following section. Meanwhile, before concluding this section, let us give a rather general sufficient condition for the assumptions of Theorem 1 to hold.

Theorem 4. Let $0<a<a_{0}$, where $a_{0}$ is defined in (3.12), and let $l>0$ be sufficiently large. Then there exists a unique solution $v$ of (3.8) for which $E[v]<0$. There is no bounded solution of (3.8), if $l>0$ is sufficiently small.

Proof. Consider the case of $l \ll 1$ first. Then it is easy to see that since the integrand in (3.14) is bounded from below uniformly in $v_{0},(3.14)$ cannot be satisfied for any $v_{0} \in\left(v_{1}, v_{2}\right)$. On the other hand, suppose now that $l \gg 1$. Then, observe that $G\left(v_{-}\right)-G(s)+s^{2}$ is a monotonically decreasing function of $s$ for $s \in\left(v_{1}, v_{2}\right)$, and increasing for $s \in\left(0, v_{1}\right)$. Therefore, for any $v_{0} \in\left(v_{1}, v_{2}\right), v_{-} \in\left(0, v_{0}\right)$ solving the second equation in (3.14), and any $s \in\left[v_{-}, v_{0}-\delta\right]$ we have 
$s^{2}-G(s) \geq\left(v_{0}-\delta\right)^{2}-G\left(v_{0}-\delta\right)>0$, provided that $\delta>0$ is sufficiently small. On the other hand

$$
G\left(v_{-}\right)-G(s)+s^{2}=2\left(\sigma_{\nu, a}\left(v_{0}\right)-v_{0}\right)\left(v_{0}-s\right)+O\left(\left(v_{0}-s\right)^{2}\right) .
$$

Therefore, the integral in (3.14) is uniformly bounded for all $v_{0} \in I \Subset\left(v_{1}, v_{2}\right)$, contradicting the assumption $l \gg 1$ for all $v_{0} \in I$, with $I$ fixed.

Therefore, the right-hand side of (3.14) may become large only when $v_{0}$ is sufficiently close to $v_{2}$ or $v_{1}$. Consider the first case first. Then we have $\sigma_{\nu, a}\left(v_{0}\right)-v_{0} \sim v_{2}-v_{0}$, and so the integral in (3.14) is dominated by the neighbourhood of $s=v_{0}$ (here and below the symbol " $\sim$ " is used in the sense of asymptotic equivalence in $C^{1}$ up to an $O(1)$ constant). It is then not difficult to show that $l \sim \ln \left(v_{2}-v_{0}\right)^{-1}$, and so for each large enough $l$ there exists a unique value of $v_{0}$ sufficiently close to $v_{2}$, such that (3.14) holds. On the other hand, if $v_{0}$ is sufficiently close to $v_{1}$, then $v_{-} \sim\left(v_{0}-v_{1}\right)^{1 /(\nu+1)}$, and the integral in (3.14) is dominated by the vicinity of $v_{-}$. A similar estimate then shows that $l \sim \ln \left(v_{0}-v_{1}\right)^{-1 /(\nu+1)}$ and, once again, there is a unique value of $v_{0}$ close enough to $v_{1}$ for which (3.14) holds for large enough $l$.

It remains to analyze the sign of $E$ for these values of $v_{0}$. If $v_{0}$ is close to $v_{1}$, then from the arguments above we have $G\left(v_{-}\right) l \sim v_{-}^{1+\nu} \ln v_{-}^{-1}$, which is asymptotically smaller than $v_{-}^{2}$ for $\nu>1$. Therefore, the third term in (3.15) dominates, and we have $E>0$ in this case. On the other hand, if $v_{0}$ is close to $v_{2}$, then both the integral and the last term in (3.15) are $O(1)$ quantities. Therefore, the second term will dominate for $l \gg 1$, and we have $E<0$.

The result of Theorem 4 appears to be quite expected, since for $l \gg 1$ front propagation in the considered problem should be governed essentially by one-dimensional traveling waves, whereas for $l \ll 1$ the strength of the positive feedback should not be sufficient to sustain a wave of signaling. It is also natural to expect that the structure of bounded solutions of (3.8) established in the proof of Theorem 4 in the case $l \gg 1$, namely, that there exist precisely two solutions of (3.8), one with positive energy and the other with negative energy, remains the same as long as (3.11) holds. Hence, by monotonic decrease of $\min E$ as a function of $l$, one would expect existence of a threshold value of $l=l_{T}>0$, such that the conclusions of Theorem 1 hold if and only if $l>l_{T}$, for every $\nu>1$ and $a<a_{0}$ fixed. Numerical evidence suggests this to be the case. Mathematically, however, the issue is to prove uniqueness of solution of (3.8) with negative energy.

\section{Examples}

We now investigate the question of uniqueness of critical points of $E$ and estimate the wave speeds in a number of particular cases. We first consider the case of the nonlinearity with the Hill coefficient $\nu=2$, corresponding to the case of moderate cooperativity in the response of the autocrine feedback. In this case from (3.13) we obtain explicitly

$$
G(v)=2\left\{v-a \arctan \left(\frac{v}{a}\right)\right\} .
$$



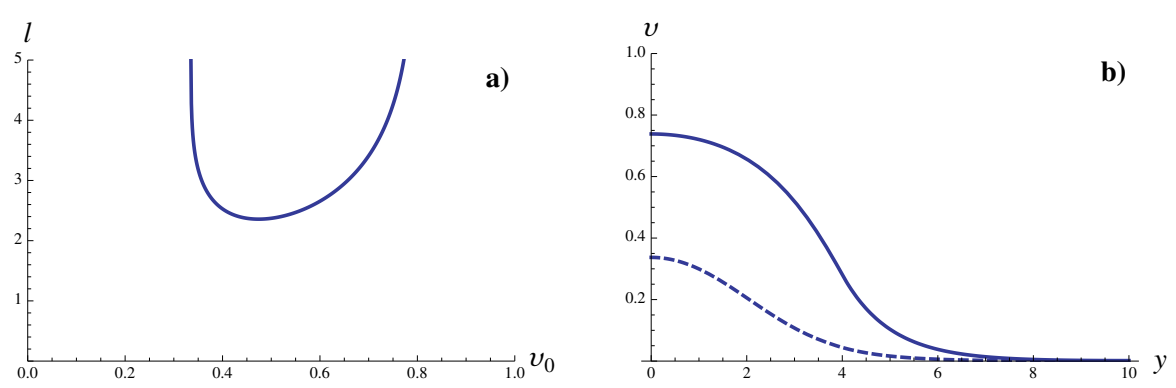

Figure 2: (a) Dependence of $l$ on $v_{0}$ when $\nu=2$ and $a=0.4$. (b) The profiles of the two bounded solutions for $\nu=2, a=0.4$ and $l=4$. The solid line shows the solution for which $E<0$.

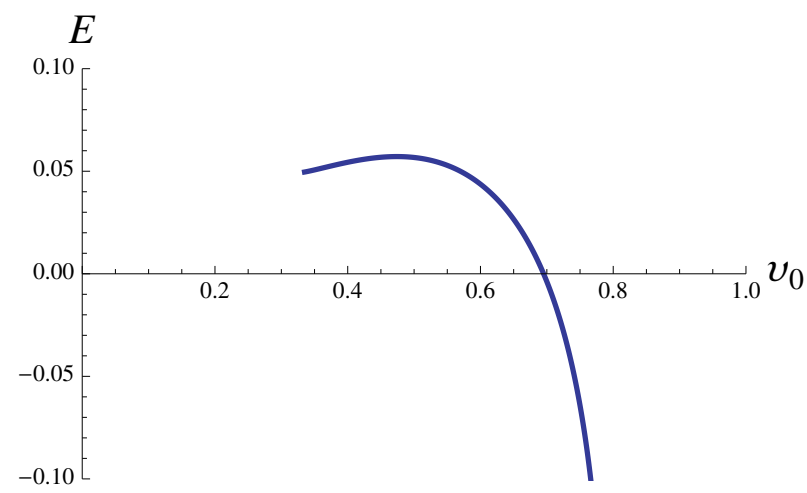

Figure 3: The energy $E$ of the bounded positive solutions as a function of $v_{0}$ for $\nu=2$ and $a=0.4$.

Also, we can explicitly calculate the value of $v_{2}$ appearing in the statement of Theorem 3 in this case. After some straightforward algebra, we find that $v_{2}=\frac{1}{2}\left(1+\sqrt{1-4 a^{2}}\right)$, provided that $a<\frac{1}{2}$. At the same time, we have $a_{0} \approx 0.4597<\frac{1}{2}$. When $a<a_{0}$, the expression $G(v)-v^{2}$ remains positive from $v=v_{2}$ down to some $v=v_{1}>0$. For example, when $a=0.4$, we have $v_{1} \approx 0.3349$ (also, $v_{2}=0.8$ for this value of $a$ ). Therefore, when $a=0.4$ and $0.335<v_{0}<0.8$, there exists a unique bounded solution of (3.8) with $v(0)=v_{0}$. We computed the values of $l$ for each $v_{0}$ from (3.14) in this interval, the result is shown in Figure 2(a). One can make several observations from these results. First, there is no positive solution of (3.8) for this value of $a$ when $l<l_{0}$, where $l_{0} \approx 2.358$. Second, when $l>l_{0}$ is fixed, there are precisely two solutions, the one corresponding to the smaller value of $v_{0}$ (Figure 2(b), dashed line), and the other corresponding to the larger value of $v_{0}$ (Figure 2(b), solid line). Note that as $l \rightarrow \infty$, the first solution approaches a homoclinic orbit possessed by (3.8) with $l=\infty$, while the second solution approaches $v=v_{2}$ as $l \rightarrow \infty$.

We also computed the energies of the solutions shown in Figure 2(a). The result, obtained by numerically integrating the expressions in (3.15) are shown in Figure 3. One can see from this figure that for each $l>l_{0}$ only the solution with the larger value of $v_{0}$ may have negative energy. This happens when $l>l_{T}$, for some $l_{T}>l_{0}$. For example, $l_{T} \approx 3.35$ when $a=0.4$.

We have simulated the onset of the traveling wave in the system with $\nu=2, a=0.4$, and $l=4$ from the initial condition $u(x, y, 0)=\operatorname{sech}^{-2}\left(0.25 \sqrt{x^{2}+y^{2}}\right)$ in a finite domain $[0,40] \times$ 


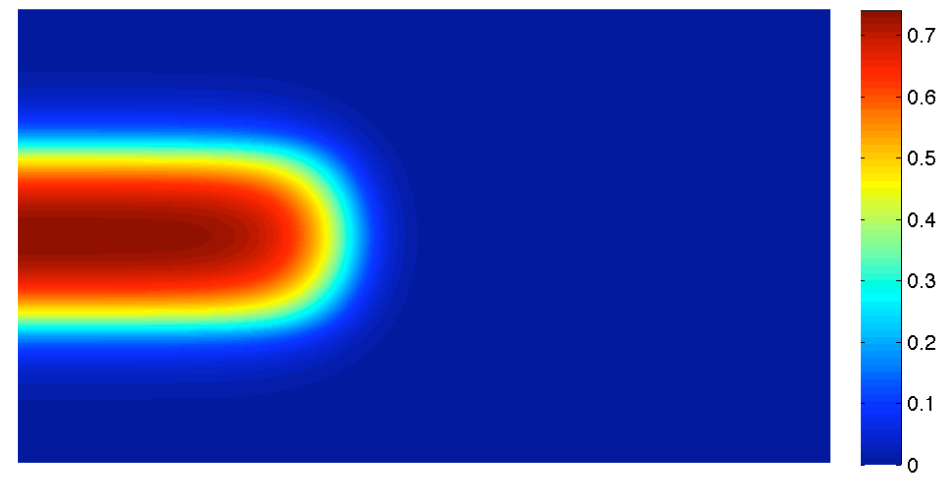

Figure 4: The colormap plot of $u$ in the wave of signaling obtained from the numerical solution of (3.2) with $\nu=2, a=0.4$ and $l=4$. Simulations were performed on a $40 \times 20$ domain with Neumann boundary conditions, using the standard finite difference discretization with $d x=0.05$.

$[-10,10]$ with Neumann boundary conditions (the effect of the boundary conditions was found to be negligible). The resulting wave profile at $t=100$ is presented in Figure 4. After an initial transient, the profile of the solution takes the shape of a "finger" advancing from left to right. It then attains a steadily moving profile with speed $c^{\dagger} \approx 0.107$. This speed is in agreement with the lower bound of $c_{\text {min }}=0.083$ obtained by using the trial function $\tilde{u}(x, y)=\frac{0.8}{\left(1+e^{x}\right)\left(1+e^{y-3.3}\right)}$ in Remark 2. On the other hand, estimating $\Phi_{c}[u]$ for all $u \in H_{c}^{1}\left(\mathbb{R}^{2}\right)$ such that $u(x, y)=u(x,-y)$, we find

$$
\begin{array}{r}
\Phi_{c}[u] \geq \int_{-\infty}^{+\infty} \int_{0}^{l} e^{c x}\left(u_{x}^{2}+u_{y}^{2}+u^{2}-G(u)\right) d y d x+\int_{-\infty}^{+\infty} e^{c x} u^{2}(x, l) d x \\
\geq \int_{-\infty}^{+\infty} \int_{0}^{l} e^{c x}\left(\left(\frac{1}{4} c^{2}+\mu_{0}+1\right) u^{2}-G(u)\right) d y d x
\end{array}
$$

where $\mu_{0}$ is the smallest eigenvalue of $-d^{2} / d y^{2}$ with boundary conditions $d v / d y=0$ at $y=0$ and $d v / d y=-v$ at $y=l$, and we applied the Poincaré inequality of [18, Lemma 2.2]. For example, for $l=4$, we have $\mu_{0} \approx 0.1$. Then, using (4.1) it is easy to see that when $a=0.4$ and $l=4$ the term in the bracket in (4.2) stays positive for all $c>c_{\max } \approx 0.45$. Note that for these parameters even a rather crude lower bound $c_{\min }$ is quite close to the actual value of $c^{\dagger}$. At the same time, since the system is near the propagation threshold at these parameters, the (also rather crude) upper bound $c_{\text {max }}$ considerably overestimates the wave speed. On the other hand, for $a=0.25$ and $l=4$, further away from the propagation threshold, by the same arguments we obtain $c_{\min } \approx 1.045$ and $c_{\text {max }} \approx 1.725$. These compare more favorably with the value of $c^{\dagger} \approx 1.15$ obtained numerically.

The second example we will consider is the case $\nu=\infty$, corresponding to strong cooperativity of the autocrine feedback. In this case we can explicitly construct all positive symmetric solution of (3.8) for $0<a<\frac{1}{2}$ (otherwise there are no solutions). After some straightforward algebra we 
find that there are exactly two solutions for each

$$
l>l_{0}=\ln (1-2 a)^{-1 / 2} .
$$

The first solution

$$
v_{\text {low }}= \begin{cases}1-\sqrt{1-2 a} \cosh y, & |y|<l_{0}, \\ a e^{l_{0}-|y|}, & |y| \geq l_{0},\end{cases}
$$

has $v_{0}=1-\sqrt{1-2 a}$ independently of $l$. Note that, compared to the case $\nu<\infty$ considered in Theorem 3, the dependence of $l$ on $v_{0}$ degenerates here. The second solution exists for all $v_{0}>1-\sqrt{1-2 a}$ when

$$
l=\ln \left(1-v_{0}\right)^{-1}>l_{0}
$$

and is given by

$$
v_{\text {high }}= \begin{cases}1-\left(1-v_{0}\right) \cosh y, & |y|<l \\ \frac{1}{2}\left(2-v_{0}\right) v_{0} e^{l-|y|}, & |y| \geq l\end{cases}
$$

Notice that we have $v_{\text {low }}<v_{\text {high }}$.

Using (3.15), we can also calculate the energy of each solution obtained above. For the first solution we obtain

$$
E\left[v_{\text {low }}\right]=a+\frac{1}{2}(1-2 a) \ln (1-2 a)>0
$$

On the other hand,

$$
E\left[v_{\text {high }}\right]=(1-2 a) \ln \left(1-v_{0}\right)+\frac{1}{2}\left(2-v_{0}\right) v_{0} .
$$

By direct inspection, the latter expression is a monotonically decreasing function of $v_{0}$ and becomes negative when $v_{0}$ is sufficiently close to 1 . Then, according to (4.5), this implies existence of a unique critical point of $E$ with negative energy for all $l>l_{T}$, for some $l_{T}=l_{T}(a)>0$, and non-existence of such a solution for $l \leq l_{T}$. Solving the obtained transcendental equations numerically, we find, e.g., that $l_{T} \approx 2.483$ for $a=0.4$.

One would naturally expect these conclusions to still hold for $\nu$ sufficiently large, but finite. Hence, for $\nu \gg 1$ the conclusions of Theorem 1 are expected to remain valid under the assumption in (3.11) only, with the asymptotic limit $v$ of the traveling wave solution at $x=-\infty$ close to $v_{\text {high }}$. We have found this agreement to be very good quantitatively already for $\nu \simeq 6$. On the other hand, the analysis for $\nu=2$ performed earlier also leads to the same conclusions regarding multiplicity of the solutions of (3.8). 


\section{Conclusions}

To summarize, we have introduced a biophysical model of autocrine relays in developing epithelia in the presence of a morphogenetic pre-pattern. We concentrated our analysis on the case in which the pre-pattern consists of a long and narrow strip of cells capable of autocrine signaling. We adapted a variational approach of [18] to establish existence of variational traveling waves, a class of traveling wave solutions relevant for signal propagation in the considered class of systems [17]. We paid particular attention to the question of multiplicity of these traveling wave solutions and showed that in all the situations that we considered, the variational traveling wave solution, if it exists, is also unique (up to translations). Thus, we established that the considered patterned autocrine relay system is a robust developmental module capable of transmitting chemical signals across large distances, with a specified transmission speed. It may be viewed as an analogue of an electrical wire for delivering electrical bits in the conventional digital electric circuits. Also, similarly to the electric circuits, more complex morphogenetic pre-patterns may be used to deliver more complicated signals across the morphogenetic field.

Our analysis of multiplicity of the traveling wave solutions, which relies on the analysis of the non-trivial steady states of the governing equation proved possible due to a particular structure of this equation in the case of a single strip capable of autocrine signaling. We took this structure into account to obtain a convenient characterization of the steady states (Theorem 3 ). This is the most novel aspect of the present analysis. As a result, we obtained easily verifiable conditions (see Theorem 4 and Sec. 4.) that allow to establish the global structure of the steady solutions and, as a consequence, of the variational traveling waves as functions of the problem parameters. Of course, these results are not unexpected for a single reaction-diffusion equation with a bistable nonlinearity localized to a strip in the plane. Yet, the particular structure of the considered model allows quantitative analytical studies of existence, multiplicity, and global bifurcations of solutions, which otherwise can be studied only via large-scale numerical simulations. It is this analysis that demonstrates the robustness of the underlying signal transmission mechanism.

Naturally, our approach also presents a trade-off between the complexity of the underlying model and the degree to which this model is amenable to analysis. Indeed, the starting point of our analysis is a model given by the system (2.1) - (2.3), which is a nonlinear system of coupled ordinary and partial differential equations. Let us emphasize that even this initial model already represents a great simplification of reality, since it lumps together many molecular species taking part in signaling, as well as ignoring all other regulatory mechanisms. Yet further simplifications leading to (2.4) were necessary to perform rigorous analysis of the model. A major challenge for mathematics in the present context is to be able to perform similar kind of analysis for more realistic models, which are always given by systems of coupled equations. Specifically, in the case of the models of autocrine relays considered in this paper the assumption of slowness of the variable $S$ compared to all other variables may not always be well-justified [22]. If this assumption is not made, but one still uses the steady-state approximation for $C$ and sets $G_{0}=0$ for simplicity, one would arrive at the following simplified model in $\mathbb{R}^{2}$ :

$$
\begin{aligned}
& u_{t}=u_{x x}+u_{y y}-u+v, \\
& v_{t}=k\left(\sigma(u) \chi_{[-l, l]}(y)-v\right),
\end{aligned}
$$


where we performed a suitable rescaling, and $k=k_{p} / k_{s}$. Now it is no longer possible to apply the variational approach of [18] to this system any more. At the same time, it turns out that the system (5.1), (5.2) falls within the category of degenerate monotone systems and, therefore, can be studied by the methods of $[26,12,27]$. Another observation, which is not immediately apparent, is that this system possesses a kind of energy that is monotonically decreasing in time. To see this, let us express $v$ in terms of $u$ through (5.1) and substitute it into (5.2), then multiply the obtained equation for $u$ by $u_{t}$ and integrate over space. After some algebra, we obtain

$$
\begin{aligned}
\frac{d H}{d t} & =-\iint_{\mathbb{R}^{2}}\left\{(1+k) u_{t}^{2} d x d y+\left|\nabla u_{t}\right|^{2}\right\} d x d y \leq 0, \\
H[u] & =\iint_{\mathbb{R}^{2}}\left\{\frac{1}{2} u_{t}^{2}+\frac{k}{2}|\nabla u|^{2}+k V(y, u)\right\} d x d y,
\end{aligned}
$$

where $V(y, u)=\int_{0}^{u}\left(s-\chi_{[-l, l]}(y) \sigma(s)\right) d s$, and $H$ is evaluated on the solutions of (5.1), (5.2). This functional can be used to establish convergence of the solutions of (5.1), (5.2) to equilibria on a finite domain, as well as to discriminate between different equilibria as possible limits for $t \rightarrow \infty$, in particular, whether a non-trivial equilibrium is reached. We note that this property remains true when $\sigma$ is replaced by a more general function, not necessarily sigmoidal, e.g. $\sigma=a^{2} u^{2} /\left(a^{4}+u^{4}\right)$ (which is peaked at $u \approx a$ ), and the system above is no longer a monotone system.

Let us mention that despite the existence of the energy $H$ in (5.4), an extension of the techniques of $[14,18]$ to $(5.1),(5.2)$ for studying propagation does not seem to be possible. The difficulty arises from the absence of diffusion in the $v$ equation and is, in fact, a common obstacle in carrying the approach of $[14,18]$ to general systems of reaction-diffusion equations. On the other hand, if one reinterprets (5.1), (5.2) as a model for two diffusible species, in which $u$ cross-activates $v$ and vice versa, then, assuming equidiffusion of $u$ and $v$ (i.e. adding a term $\Delta v$ to (5.2)), it is again possible to derive a variational characterization of propagation and traveling waves, as before. The computation is rather tedious, but the functional $\Phi_{c}$ whose critical points are the traveling wave solutions now is:

$$
\begin{gathered}
\Phi_{c}[u]=\iint_{\mathbb{R}^{2}} e^{c x}\left\{\frac{1}{2}\left(u_{x x}+u_{y y}\right)^{2}+\frac{1}{2}(1+k)\left(u_{x}^{2}+u_{y}^{2}\right)\right. \\
\left.+\frac{1}{2} c^{2} u_{y}^{2}+k V(y, u)\right\} d x d y .
\end{gathered}
$$

It should then be possible to study the non-trivial minimizers of this functional following Refs. $[14,18]$. In conclusion, analysis of systems of reaction-diffusion equations arising from the models of developmental pattern formation still remains a significant mathematical challenge. Here the use of variational methods may show some promise.

Note added in proof. After this paper was accepted for publication, we became aware of some related results for a similar class of problems $[5,6]$. 


\section{Acknowledgements}

This work was supported by NSF via grants DMS-0718027 (CBM) and DMS-0718604 (SYS).

\section{References}

[1] D. G. Aronson, H. F. Weinberger. Multidimensional diffusion arising in population genetics. Adv. Math., 30 (1978), 33-58.

[2] H. L. Ashe, J. Briscoe. The interpretation of morphogen gradients. Development, 133 (2006), 385-394.

[3] H. Berestycki, F. Hamel. Generalized travelling waves for reaction-diffusion equations. Perspectives in nonlinear partial differential equations, volume 446 of Contemp. Math., pages 101-123. Amer. Math. Soc., Providence, RI, 2007.

[4] J. D. Buckmaster, G. S. S. Ludford. Theory of laminar flames. Cambridge University Press, Cambridge, 1982.

[5] G. Chapuisat. Existence and nonexistence of curved front solution of a biological equation. J. Differential Equations 236 (2007), 237-279.

[6] G. Chapuisat and R. Joly, Asymptotic profiles for a travelling front solution of a biological equation. Preprint.

[7] G. Dal Maso. An Introduction to Г-Convergence. Birkhäuser, Boston, 1993.

[8] P. C. Fife. Mathematical Aspects of Reacting and Diffusing Systems. Springer-Verlag, Berlin, 1979.

[9] M. Freeman. Feedback control of intercellular signalling in development. Nature, 408 (2000), 313-319.

[10] D. Gilbarg, N. S. Trudinger. Elliptic Partial Differential Equations of Second Order. SpringerVerlag, Berlin, 1983.

[11] U. Heberlin, K. Moses. Mechanisms of Drosophila retinal morphogenesis: the virtues of being progressive. Cell, 81 (1995), 987-990.

[12] B. Kazmierczak, V. Volpert. Travelling calcium waves in systems with non-diffusing buffers. Math. Models Methods Appl. Sci., 18 (2008), 883-912.

[13] J. Lembong, N. Yakoby, S. Y. Shvartsman. Pattern formation by dynamically interacting network motifs. Proc. Natl. Acad. Sci. USA, 106 (2009), 3213-3218. 
[14] M. Lucia, C. B. Muratov, M. Novaga. Existence of traveling waves of invasion for GinzburgLandau-type problems in infinite cylinders. Arch. Rational Mech. Anal., 188 (2008), 475508.

[15] A. Lunardi. Analytic semigroups and optimal regularity in parabolic problems, volume 16 of Progress in Nonlinear Differential Equations and their Applications. Birkhäuser, Basel, 1995.

[16] A. Martinez-Arias, A. Stewart. Molecular principles of animal development. Oxford University Press, New York, 2002.

[17] C. B. Muratov. A global variational structure and propagation of disturbances in reactiondiffusion systems of gradient type. Discrete Cont. Dyn. S., Ser. B, 4 (2004), 867-892.

[18] C. B. Muratov, M. Novaga. Front propagation in infinite cylinders. I. A variational approach. Comm. Math. Sci., 6 (2008), 799-826.

[19] C. B. Muratov, F. Posta, S. Y. Shvartsman. Autocrine signal transmission with extracellular ligand degradation. Phys. Biol., 6 (2009), 016006.

[20] C. B. Muratov, S. Y. Shvartsman. Signal propagation and failure in discrete autocrine relays. Phys. Rev. Lett., 93 (2004), 118101 pp. 1-4.

[21] M. Přibyl, C. B. Muratov, S. Y. Shvartsman. Discrete models of autocrine cell communication in epithelial layers. Biophys. J., 84 (2003), 3624-3635.

[22] M. Přibyl, C. B. Muratov, S. Y. Shvartsman. Long-range signal transmission in autocrine relays. Biophys. J., 84 (2003), 883-896.

[23] N. Shigesada, K. Kawasaki. Biological invasions: theory and practice. Oxford Series in Ecology and Evolution. Oxford Univ. Press, Oxford, 1997.

[24] T. Tabata, Y. Takei. Morphogens, their identification and regulation. Development, 131 (2004), 703-712.

[25] J. J. Tyson, K. Chen, B. Novak. Network dynamics and cell physiology. Nat. Rev. Mol. Cell Biol., 2 (2001), 908-916.

[26] A. I. Volpert, V. A. Volpert, V. A. Volpert. Traveling wave solutions of parabolic systems. AMS, Providence, 1994.

[27] V. Volpert, A. Volpert. Existence of multidimensional travelling waves in the bistable case. C. R. Acad. Sci. Paris Sér. I Math., 328 (1999), 245-250.

[28] H. S. Wiley, S. Y. Shvartsman, D. A. Lauffenburger. Computational modeling of the EGFreceptor system: a paradigm for systems biology. Trends Cell Biol., 13 (2003), 43-50.

[29] L. Wolpert, R. Beddington, T. Jessel, P. Lawrence, E. Meyerowitz. Principles of Development. Oxford University Press, Oxford, 1998. 
[30] J. Xin. Front propagation in heterogeneous media. SIAM Review, 42 (2000), 161-230.

[31] N. Yakoby, J. Lembong, T. Schüpbach, S. Y. Shvartsman, Drosophila eggshell is patterned by sequential action of feedforward and feedback loops. Development, 135 (2008), 343-351. 